J. Lake Sci.(湖泊科学), 2019, 31(1):220-235

DOI 10. 18307/2019. 0121

(c) 2019 by Journal of Lake Sciences

\title{
一株淡水红藻的形态及分子系统发育研究
}

\author{
方輢鹏, 南芳茹, 冯佳, 吕俊平, 刘琪, 谢树莲** \\ ( 山西大学生命科学学院,太原 030006 )
}

\begin{abstract}
摘 要: 以采自山西晋祠泉的一株淡水红藻 JC1712001 为实验材料, 对其进行分子系统发育分析和形态特征鉴定, 同时 利用分子数据对其进行生物地理学研究. 结果显示, 该株淡水红藻 JC1712001 与美国、西班牙地区报道的马赫拉熊野澡 (Kumanoa mahlacensis Kumano) 遗传距离最近, 基于 $r b c \mathrm{~L} 、 \mathrm{COI}$ 和 UPA 序列的遗传距离均为 0 . 基于 3 个序列,利用贝叶斯 法、最大似然法和邻接法 3 种方法构建的系统发育树具有高度一致的系统发育关系, JC1712001 均与西班牙及美国地区 已报道的马赫拉熊野藻聚为一支, 支持率都在 $97 \%$ 以上, 支持其为马赫拉熊野藻, 同时也表明 3 个基因的高度保守性. 形 态特征的观察也支持这个结果. 马赫拉熊野藻在欧洲、亚洲、北美洲均有分布, RASP 软件构建的地理起源图谱显示, 欧洲 类群地理起源更为古老. 马赫拉熊野藻为稀有种,本文的研究结果丰富了我国淡水红藻的分布,为其系统发育和地理起 源研究提供了依据.
\end{abstract}

关键词: 马赫拉熊野藻; $r b c \mathrm{~L} ; \mathrm{COI} ; \mathrm{UPA}$; 系统发育;地理起源

\section{Morphology and molecular phylogeny of a freshwater red algae}

FANG Kunpeng, NAN Fangru, FENG Jia, LV Junping, LIU Qi \& XIE Shulian **

( School of Life Science, Shanxi University, Taiyuan 030006, P.R.China)

Abstract: The molecular analysis and morphological identification of a freshwater red alga JC1712001, collected from Jinci spring, Shanxi Province, North China, were performed, and its biogeographic analysis was conducted using molecular biological evidence. The results showed that the genetic distances between JC1712001 and Kumanoa mahlacensis Kumano strains from the United States and Spanish were all 0 based on the $r b c \mathrm{~L}$, COI and UPA sequences, indicating that the three genes were highly conserved. Phylogenetic trees based on three sequences by three methods, including Bayesian method, maximum likelihood method and neighboring method, were highly consistent. In the phylogenetic trees, JC1712001 were clustered with K. mahlensis from Spain and the United States, and the support rates were all above 97\%. According to molecular biological evidence, JC1712001 is also classified as $K$. mahlensis. The geographical origin map constructed by RASP software showed that K. mahlensis was distributed in Europe, Asia, and North America, and the origin of European group is more ancient. K. mahlensis is a rare species, and the results of this study have enriched the distribution of freshwater red algae in China. Meanwhile, it also provided data for the phylogenetic analysis and the geographical origin research.

Keywords: Kumanoa mahlacensis; rbcL; COI; UPA; phylogenetic analysis; geographical origin

淡水红藻缺乏鞭毛,有叶绿素 $\mathrm{a}$ 和藻胆蛋白, 以红藻淀粉作为储存物质, 同时类囊体以单层的形式出现 在叶绿体中, 是最古老的真核生物类群之一 ${ }^{[1]}$. 与海洋红藻相比,淡水红藻由于生境要求特殊, 种类较少, 种 群较小,多数种类分布罕见 ${ }^{[2]}$.

在淡水红藻中, 串珠藻科 Batrachospermaceae 约有 150 种, 是种类最多、研究最活跃的一个类群 ${ }^{[3]}$. 串珠 藻科 Batrachospermaceae 是由 Agardh 在 1824 年建立的 ${ }^{[4]}$, 但其包含的属种研究历史则更长. Roth 于 1797 年 建立了串珠藻属 Batrachospermum ${ }^{[5]}$. 随后, 1820 年, Charospermum 被建立 ${ }^{[6]}$, 仅含 1 种, C. glomeratum Link,

* 国家自然科学基金项目 (31670208,31370239) 和山西省教育厅 1331 工程支持项目联合资助. 2018-06-06 收稿; 2018-07-04 收修改稿. 方鵾鹏( 1993 ）, 男,博士研究生; E-mail：1349303542@ qq.com.

** 通信作者;E-mail: xiesl@ sxu.edu.cn. 
但该属并未得到后来学者的认可,所含的 1 种也已被作为串珠藻属胶串珠藻 B. gelatinosum Linnaeus 的异名. 1828 年, Bonnemaison 建立了 Torularia, 包含两个种, T. dillenii Bonnemaison 和 T. lenta Bonnemaison ${ }^{[7]}$, 同样, 该属也未得到后来学者的认可,所辖的两种都已被作为 Setacea atra 的异名 ${ }^{[8]}$. Harvey 于 1858 年基于藻体皮 层紧密、初生枝细胞明显膨大、分枝密集、受精丝直角状弯曲、果孢子体生于中轴和皮层间的凹穴处、果孢子 囊萌发不形成丝状体而是产生一团未分化的薄壁细胞等特征建立了托氏藻属 Tuomeya, 目前该属含 1 种 ${ }^{[9]}$. Kylin 于 1912 年基于果胞基部不对称和果孢子体不定形建立了连珠藻属 Sirodotia, 目前该属含 10 种 ${ }^{[10]}$. Skuja 于 1934 年依据皮层较紧密、受精丝明显向一侧弯曲和不具有定形的果孢子体建立了假枝藻属 Nothocladus, 目前该属含 17 种 ${ }^{[11]}$. Entwisle 于 1989 年在澳大利亚东南部溪流中发现了一株红藻, 藻体较硬, 圆柱 形, 结节状, 具有松散排列的髓丝以及外皮层, 但未见生殖结构, 据此建立了裸管藻属 Psilosiphon, 含 1 种 ${ }^{[12]}$. 进人本世纪, 分子生物学手段成为串珠藻科分类和系统发育研究的主要手段. Saunders 和 Necchi 于 2002 年 建立了 Balliopsis, 主要是基于核糖体 SSU 和部分 LSU 序列的差异, 目前该属含 2 种 ${ }^{[13]} .2007$ 年, 基于 LSU 和 $r b c \mathrm{~L}$ 序列 Petrohua 被建立, 目前该属含 1 种 ${ }^{[14]}$. Entwisle 于 2009 年基于分子数据建立了熊野藻属 Kumanoa, 目前该属含 46 种 $^{\left[{ }^{[3]}\right.}$. 之后, 基于分子数据先后建立西斯藻属 Sheathia (目前含 10 种 $)^{[15]}$ 、Setacea ( 现已被作 为 Atrophycus 的异名 $)^{[16]} 、$ Nocturama $(\text { 目前含 } 1 \text { 种 })^{[17]} 、$ Atrophycus $(\text { 目前含 } 3 \text { 种 })^{[16]}$ 、Lympha $(\text { 目前含 } 1 \text { 种 })^{[18]}$ 和飞跃藻属Volatus $\left(\right.$ 目前含 3 种 ${ }^{[19]}$ 等.

由此可见,运用分子数据阐释串珠藻科的进化关系已成为极为活跃的领域,在越来越多类群被发现的 同时,也导致出现了更为复杂的分类系统, 单纯依赖形态特征鉴定物种已不能满足研究要求 ${ }^{[13-19]}$.

作者于 2017 年 11 月在山西晋祠泉采到一株串珠藻状的植物. 为了对其准确鉴定, 本研究对这株藻体 进行了形态结构的观察, 并测定了其 $r b c \mathrm{~L} 、 \mathrm{COI}$ 和 UPA 基因序列, 构建了系统发育树, 进行了系统发育分析, 在此基础上基于 $r b c \mathrm{~L}$ 序列构建了其地理系统发育图谱, 以期确定该株植物的分类地位, 并阐明与其亲缘种 之间的系统发育关系.

\section{1 材料和方法}

\section{1 实验材料}

实验所用藻株 JC1712001 采自山西晋祠泉 $\left(37^{\circ} 42^{\prime} \mathrm{N}, 112^{\circ} 26^{\prime} \mathrm{E}\right)$, 生长在出水口下游 $5 \sim 6 \mathrm{~m}$, 流速较缓的近 岸处, 11 月至翌年 2 月生长较旺盛. 水温 $16.0^{\circ} \mathrm{C}$, 电导率 $1149 \mathrm{~S} / \mathrm{m}$, 可溶性固体质量浓度 $1.023 \%, \mathrm{pH} 7.26$.

藻体经无菌水清洗去除杂质, 显微镜下观察并去除具有明显杂藻污染的藻枝, 处理后的藻体经液氮速 冻后 $-80^{\circ} \mathrm{C}$ 保存, 用于分子鉴定. 取部分藻体供形态学观察, 并用 $4 \%$ 的甲醛溶液固定 ${ }^{[20]}$. 保存藻体标本于 山西大学植物标本馆(SXU), 标本号 SXU-SAS17031.

\section{2 分子鉴定}

1.2.1 总 DNA 提取 使用植物组织 DNA 抽提试剂盒 (Dzup (Plant) Genomic DNA Isolation Reagent, 上海生工 生物工程), 按照使用说明进行 DNA 提取.

1.2.2 目的基因扩增及序列测定 查阅文献 ${ }^{[21-23]}$, 确定合适的 PCR 体系及引物, $r b c \mathrm{~L}$ 基因引物 F160: CCTCAACCAGGAGTAGATCC 和 rbcLr: ACATTTGCTGTTGGAGTCTC, COI 基因引物 GazF1 : TCAACAAATCATAAAGATATTGG 和 GazR1: ACTTCTGGATGTCCAAAAAAYCA, UPA 基因引物 p23SrV-f1: GGACAGAAAGACCCTATGAA 和 p $23 \mathrm{SrV}$-r1 : TCAGCCTGTTATCCCTAGAG; 反应体系均为 $20 \mu \mathrm{l}$, 包括 $0.5 \mu \mathrm{l}$ DNA 模板、正向和 反向引物各 $2 \mu \mathrm{l} 、 2 \mu \mathrm{l}$ dNTPs、2 $\mu \mathrm{l} 10 \times$ buffer $\left(\mathrm{Mg}^{2+}\right)$ 及 $0.2 \mu \mathrm{l}$ Taq DNA 聚合酶(大连宝生物公司 TaKaRa). 扩增 $r b c \mathrm{~L} 、 \mathrm{COI}$ 和 UPA 基因序列, PCR 产物经 $1 \%$ 的琼脂糖凝胶电泳检测 (电压 $120 \mathrm{~V}$, 时间 $20 \mathrm{~min}$ ), 产物由 北京华大公司采用 Sanger 双脱氧链终止法进行双向测序 (华大基因有限公司, 北京).

1.2.3 系统发育分析 将获得的 $r b c \mathrm{~L} 、 \mathrm{COI}$ 和 UPA 基因序列用 NCBI (https://www.ncbi.nlm. nih.gov/) 中的 blast 工具与 GenBank 已发表的相应序列进行在线比对, 根据比对结果选取相关基因序列 (表 1), 对所选序 列利用 Clustal X 软件进行分析, 经校正的序列用于后续系统发育分析. 对下载序列和所测样本序列用 MEGA 6.0 分析其序列特征, 确定其序列可变异位点和简约位点, 计算各近缘种的遗传距离, 并采用邻接法 ( NJ)、最大似然法 (ML) 和贝叶斯法 (BA) 分别构建 3 个基因的系统发育树. 
表 1 GenBank 中下载的供分析的 $r b c \mathrm{~L} 、 \mathrm{COI}$ 和 UPA 序列及登录号

Tab.1 GenBank accession number of related $r b c \mathrm{~L}$, COI and UPA gene sequences in this study

\begin{tabular}{|c|c|c|c|c|c|}
\hline 分类单位 & 样本 & $r b c \mathrm{~L}$ & COI & UPA & 参考文献 \\
\hline \multirow[t]{5}{*}{ Atrophycus puiggarianus } & BR09122001a & FJ386462 & - & - & {$[3]$} \\
\hline & RPD & KP203886 & - & - & {$[24]$} \\
\hline & Sp8 & - & KX703035 & - & {$[8]$} \\
\hline & PEVV4 & - & KT894751 & - & {$[8]$} \\
\hline & CAMB & - & KT894742 & - & 未出版 \\
\hline \multirow[t]{3}{*}{ Bangia atropurpurea } & sxqy & KJ023703 & - & - & {$[25]$} \\
\hline & JB328 & - & DQ191330 & - & 未出版 \\
\hline & G00033 & - & - & EF426661 & {$[23]$} \\
\hline \multirow[t]{2}{*}{ B. fuscopurpurea } & GWS001869 & JN028931 & JN028460 & - & {$[26]$} \\
\hline & ARS03783 & - & - & HQ421200 & {$[27]$} \\
\hline \multirow[t]{7}{*}{ Batrachospermum gelatinosum } & MA140_07 & GU810833 & - & - & {$[28]$} \\
\hline & МICH & EF375888 & - & - & {$[29]$} \\
\hline & 12 & - & EU636743 & EU628669 & {$[30]$} \\
\hline & JR-10-1 & - & HQ412539 & - & {$[31]$} \\
\hline & FR2 & - & GU810827 & - & {$[30]$} \\
\hline & BHO :A-0096 & - & - & KY225795 & 未出版 \\
\hline & - & - & - & KJ143749 & 未出版 \\
\hline \multirow[t]{4}{*}{ B. guianense } & clone FG8 & AY423396 & - & - & {$[32]$} \\
\hline & 5 & - & EU095968 & - & {$[30]$} \\
\hline & 14 & - & EU636713 & - & {$[30]$} \\
\hline & 15 & - & - & EU628663 & {$[33]$} \\
\hline \multirow[t]{8}{*}{ B. helminthosum } & Bhelst05 & KJ825955 & - & - & {$[34]$} \\
\hline & - & AB114643 & - & - & {$[35]$} \\
\hline & clone ct3rbcl & AF244113 & - & - & {$[36]$} \\
\hline & 0 & - & EU073847 & - & {$[30]$} \\
\hline & 23 & - & EU636733 & - & {$[33]$} \\
\hline & haplotype 2 & - & - & EU073850 & {$[30]$} \\
\hline & haplotype 3 & - & - & EU073851 & {$[30]$} \\
\hline & haplotype 4 & - & - & EU073852 & {$[30]$} \\
\hline \multirow[t]{7}{*}{ B. macrosporum } & 11 clone BR3 & EU106058 & - & - & {$[37]$} \\
\hline & BHO : MR22 & KF557567 & - & - & {$[38]$} \\
\hline & 96 & - & EU095975 & - & {$[30]$} \\
\hline & 45 & - & EU636747 & - & {$[33]$} \\
\hline & 37 & - & - & EU628676 & {$[33]$} \\
\hline & 53 & - & - & EU628675 & {$[33]$} \\
\hline & 43 & - & - & EU628673 & {$[33]$} \\
\hline \multirow[t]{7}{*}{ B. turfosum } & UTEX 1494 & DQ523254 & - & - & {$[39]$} \\
\hline & Bturst18 & KJ825963 & - & - & [39] \\
\hline & GDA-A 8134 & - & KM592950 & - & 未出版 \\
\hline & 22 & - & EU095972 & - & {$[30]$} \\
\hline & BHO :A-0132 & - & - & KY225796 & 未出版 \\
\hline & 62 & - & - & EU628651 & {$[33]$} \\
\hline & 61 & - & - & EU628650 & {$[33]$} \\
\hline \multirow[t]{2}{*}{ Kumanoa abilii } & $\mathrm{C} 1$ & GQ368882 & - & - & {$[24]$} \\
\hline & $\mathrm{C} 7$ & GQ368883 & - & - & {$[24]$} \\
\hline
\end{tabular}


续表 1

\begin{tabular}{|c|c|c|c|c|c|}
\hline 分类单位 & 样本 & $r b c \mathrm{~L}$ & COI & UPA & 参考文献 \\
\hline & KabiliiC7 & - & JN604915 & JN602670 & {$[40]$} \\
\hline \multirow[t]{9}{*}{ K. ambigua } & Bambig & AF209988 & - & - & [41] \\
\hline & 35 & GQ368885 & - & - & [24] \\
\hline & 13 & - & EU095970 & - & {$[30]$} \\
\hline & 3 & - & EU636723 & - & {$[33]$} \\
\hline & clone FG13 & AY423391 & - & - & [32] \\
\hline & MICH & EF452238 & - & - & [32] \\
\hline & 2 & - & - & EU628652 & [33] \\
\hline & 3 & - & - & EU628653 & {$[33]$} \\
\hline & Kambigua35 & - & - & JN602672 & {$[40]$} \\
\hline \multirow[t]{2}{*}{ K. americana } & KamerMS302 & JN589996 & - & - & {$[40]$} \\
\hline & KamBigCkLA & - & JN604910 & JN602665 & {$[40]$} \\
\hline \multirow[t]{4}{*}{ K. australica } & KausTJE3376 & JN590000 & - & - & {$[40]$} \\
\hline & TJE2699 & FJ386463 & - & - & {$[3]$} \\
\hline & 4 & - & EU636719 & EU628654 & {$[33]$} \\
\hline & 5 & - & EU636720 & EU628655 & {$[33]$} \\
\hline \multirow[t]{2}{*}{ K. breviarticulata } & AM2 & GQ368886 & - & - & [24] \\
\hline & 7 & - & EU636718 & EU628658 & {$[33]$} \\
\hline \multirow[t]{2}{*}{ K. capensis } & SJRP :PNCV & JX504697 & JX504695 & JX504699 & {$[40]$} \\
\hline & SJRP : CAN & JX504698 & JX504696 & JX504700 & {$[40]$} \\
\hline \multirow[t]{4}{*}{ K. cipoensis } & C11 & GQ368887 & - & - & {$[24]$} \\
\hline & C15 & GQ368888 & - & - & {$[24]$} \\
\hline & KcipensisC15 & - & JN604919 & JN602674 & {$[40]$} \\
\hline & KcipensisC11 & - & JN604918 & JN602673 & {$[40]$} \\
\hline \multirow[t]{3}{*}{ K. curvata } & Kcurvatasp65 & JN590012 & JN604925 & - & {$[40]$} \\
\hline & SAS08041 & KC511077 & KC511073 & - & {$[42]$} \\
\hline & - & - & - & KJ143745 & {$[43]$} \\
\hline \multirow[t]{3}{*}{ K. deminuta } & Bdemin & AF209981 & - & - & {$[41]$} \\
\hline & 37 & - & EU095973 & - & {$[30]$} \\
\hline & 10 & - & - & EU628660 & [33] \\
\hline \multirow[t]{3}{*}{ K. equisetoidea } & AM1.2 & GQ368889 & - & - & {$[24]$} \\
\hline & AM14 & GQ368890 & - & - & {$[24]$} \\
\hline & 11 & - & EU636716 & EU628659 & {$[33]$} \\
\hline \multirow[t]{2}{*}{ K. faroensis } & Kfar_OH & JN590001 & - & JN602662 & {$[40]$} \\
\hline & KfaroSF01 & - & JN604907 & - & {$[40]$} \\
\hline \multirow[t]{2}{*}{ K. globospora } & В014 & GQ368891 & - & - & {$[24]$} \\
\hline & BglobBO14 & - & JN604923 & JN602678 & {$[40]$} \\
\hline \multirow[t]{4}{*}{ K. gracillima } & clone FG10 & AY423395 & - & - & {$[32]$} \\
\hline & KgracillDEL & JN590013 & - & - & {$[40]$} \\
\hline & KgracFG10 & - & JN604913 & JN602668 & {$[40]$} \\
\hline & Kgracill_Del & - & JN604927 & JN602683 & {$[40]$} \\
\hline \multirow[t]{2}{*}{ K. gudjewga } & KgudjewTJE3370 & JN590002 & JN604908 & JN602663 & {$[40]$} \\
\hline & KgudjewTJE3374 & JN590003 & JN604909 & JN602664 & {$[40]$} \\
\hline \multirow[t]{2}{*}{ K. holtonii } & KholCrippleAL & - & - & JN602676 & {$[40]$} \\
\hline & KholCCAL & JN590004 & JN604921 & - & {$[40]$} \\
\hline K. intorta & clone FG17 & AY423397 & - & - & {$[32]$} \\
\hline
\end{tabular}


续表 1

\begin{tabular}{|c|c|c|c|c|c|}
\hline 分类单位 & 样本 & $r b c \mathrm{~L}$ & $\mathrm{COI}$ & UPA & 参考文献 \\
\hline & SAS06218 & JQ028695 & - & - & {$[44]$} \\
\hline & 11 & - & EU095969 & - & {$[30]$} \\
\hline & SAS06020 & - & KC511074 & - & {$[42]$} \\
\hline & - & - & - & KJ143744 & {$[43]$} \\
\hline & 33 & - & EU636717 & EU628657 & {$[33]$} \\
\hline \multirow[t]{2}{*}{ K. iriomotensis } & KiriomotSAWC & JN590011 & - & - & {$[40]$} \\
\hline & KspSA & - & - & JN602681 & {$[40]$} \\
\hline \multirow[t]{3}{*}{ K. louisianae } & - & AF029144 & - & - & {$[21]$} \\
\hline & BlouisMS2 & - & JN604924 & - & {$[40]$} \\
\hline & BlouisMS2 & JN590005 & - & JN602679 & {$[40]$} \\
\hline \multirow[t]{4}{*}{ K. mahlacensis } & MUB-ALGAS & JQ713334 & - & - & {$[45]$} \\
\hline & JC1712001 & MH220972 & MH220973 & MH220972 & 本研究 \\
\hline & Kmah_NM & JN590006 & JN604906 & JN602661 & {$[40]$} \\
\hline & Kmah_TX & JN590007 & JN604905 & JN602660 & {$[40]$} \\
\hline \multirow[t]{4}{*}{ K. nodiflora } & clone FG8 & AY423398 & - & - & {$[32]$} \\
\hline & clone FG12 & AY423399 & - & - & {$[32]$} \\
\hline & 57 & - & EU636714 & EU628661 & {$[33]$} \\
\hline & 58 & - & EU636715 & EU628662 & {$[33]$} \\
\hline \multirow[t]{3}{*}{ K. procarpum } & BR13032007 & FJ386464 & - & - & {$[3]$} \\
\hline & Kprocarpa & - & JN604920 & JN602675 & {$[40]$} \\
\hline & - & - & - & KY225798 & 未出版 \\
\hline K. skujana & KskuSuckSC & JN590008 & JN604922 & JN602677 & {$[40]$} \\
\hline \multirow[t]{4}{*}{ K. spermatiophora } & 00508-00001 & KC596190 & - & - & {$[46]$} \\
\hline & - & AY424882 & - & - & 未出版 \\
\hline & 00508 & - & - & EF426659 & {$[23]$} \\
\hline & 59 & - & EU636722 & EU628656 & {$[33]$} \\
\hline K. tabagatenensis & KtabPalau & JN590009 & JN604914 & JN602669 & {$[40]$} \\
\hline K. vittata & KvittTJE3371 & JN590010 & - & JN602666 & {$[40]$} \\
\hline \multirow[t]{8}{*}{ Sheathia arcuata } & Bulg2_06 & GU457344 & - & - & {$[47]$} \\
\hline & SAS090318 & JN086522 & - & - & {$[48]$} \\
\hline & 04952-00001 & - & KC596305 & - & {$[46]$} \\
\hline & GDA-A 6432 & - & KM592947 & - & 未出版 \\
\hline & $\mathrm{TW}-10$ & - & JX669681 & - & 未出版 \\
\hline & 07238-00001 & - & - & KC596212 & {$[46]$} \\
\hline & 00878-00001 & - & - & KC596192 & {$[46]$} \\
\hline & - & - & - & KJ143746 & {$[42]$} \\
\hline
\end{tabular}

\section{3 形态观察}

在光学显微镜( OLYMPUS BX51 with DP 72, 日本)下观察藻体形态结构并采集图像.

\section{4 地理起源分析}

对 GenBank 中获得的 $r b c \mathrm{~L}$ 序列进行篎选, 保留每个物种中不同地理来源的序列, 同一物种中地理位置 相近的序列仅保留一条, 并按地理位置 A-南美洲、B-北美洲、C-大洋洲、D-欧洲、E-亚洲、F-太平洋岛国和 G-非洲进行分类 (表 2). 结合贝叶斯系统树结果, 运用 RASP 软件构建近缘物种祖先地理分布图. 
表 2 GenBank 中下载的供分析的 $r b c \mathrm{~L}$ 序列及登录号

Tab.2 GenBank accession number of related $r b c \mathrm{~L}$ gene sequences in this study

\begin{tabular}{|c|c|c|c|}
\hline 分类单位 & 序列登录号 & 采集地 & 参考文献 \\
\hline \multirow[t]{2}{*}{ Atrophycus puiggarianus } & KP203886 & A 巴西 & {$[24]$} \\
\hline & FJ386462 & A 巴西 & {$[3]$} \\
\hline Bangia atropurpurea & KJ023703 & E 中国 & {$[25]$} \\
\hline \multirow[t]{2}{*}{ Batrachospermum gelatinosum } & GU810833 & B 美国 & {$[28]$} \\
\hline & EF375888 & D 英国 & {$[29]$} \\
\hline B. guianense & AY423396 & A 法属圭亚那(法国) & {$[32]$} \\
\hline \multirow[t]{3}{*}{ B. helminthosum } & KJ825955 & D 法国 & {$[34]$} \\
\hline & AB114643 & $\mathrm{E}$ 日本 & {$[35]$} \\
\hline & AF244113 & B 美国 & {$[36]$} \\
\hline \multirow{2}{*}{ B. macrosporum } & EU106058 & B 美国 & {$[37]$} \\
\hline & KF557567 & $\mathrm{E}$ 马来西亚 & {$[38]$} \\
\hline B. turfosum & DQ523254 & D 法国 & {$[39]$} \\
\hline \multirow[t]{3}{*}{ Kumanoa ambigua } & AF209988 & C 澳大利亚 & {$[41]$} \\
\hline & AY423391 & A 法属圭亚那(法国) & {$[32]$} \\
\hline & GQ368885 & A 巴西 & {$[24]$} \\
\hline K. deminuta & AF209981 & C 澳大利亚 & {$[41]$} \\
\hline K. gracillima & JN590013 & A 法属圭亚那(法国) & {$[40]$} \\
\hline \multirow[t]{2}{*}{ K. intorta } & AY423397 & A 法属圭亚那(法国) & {$[32]$} \\
\hline & JQ028695 & $\mathrm{E}$ 中国 & {$[45]$} \\
\hline \multirow[t]{2}{*}{ K. louisianae } & JN590005 & B 美国 & {$[40]$} \\
\hline & AF029144 & B 美国 & {$[21]$} \\
\hline K. nodiflorum & AY423398 & A 法属圭亚那(法国) & {$[32]$} \\
\hline \multirow[t]{2}{*}{ K. spermatiophorum } & AY424882 & F 夏威夷( 美国) & 未出版 \\
\hline & KC596190 & B 美国 & {$[46]$} \\
\hline K. abilii & GQ368882 & A 巴西 & {$[24]$} \\
\hline K. americana & JN589996 & B 美国 & {$[40]$} \\
\hline \multirow[t]{2}{*}{ K. australica } & JN590000 & C 澳大利亚 & {$[40]$} \\
\hline & FJ386463 & A 巴西 & {$[3]$} \\
\hline K. breviarticulata & GQ368886 & A 巴西 & {$[24]$} \\
\hline K. capensis & JX504698 & A 巴西 & {$[40]$} \\
\hline K. cipoensis & GQ368888 & A 巴西 & {$[24]$} \\
\hline \multirow[t]{2}{*}{ K. curvata } & JN590012 & A 巴西 & {$[40]$} \\
\hline & KC511077 & E 中国 & {$[42]$} \\
\hline K. equisetoidea & GQ368890 & A 巴西 & {$[24]$} \\
\hline K. faroensis & JN590001 & B 美国 & {$[40]$} \\
\hline K. globospora & GQ368891 & A 巴西 & {$[24]$} \\
\hline K. gudjewga & JN590003 & $\mathrm{C}$ 澳大利亚 & {$[40]$} \\
\hline K. holtonii & JN590004 & B 美国 & {$[40]$} \\
\hline K. iriomotensis & JN590011 & $\mathrm{G}$ 南非 & {$[40]$} \\
\hline \multirow[t]{3}{*}{ K. mahlacensis } & JQ713334 & D 西班牙 & {$[44]$} \\
\hline & MH220972 & $\mathrm{E}$ 中国 & 本研究 \\
\hline & JN590006 & B 美国 & {$[40]$} \\
\hline K. procarpum & FJ386464 & A 巴西 & {$[3]$} \\
\hline K. skujana & JN590008 & B 美国 & {$[40]$} \\
\hline K. tabagatenensis & JN590009 & C 帕劳 & {$[40]$} \\
\hline K. vittata & JN590010 & C 澳大利亚 & {$[40]$} \\
\hline \multirow[t]{2}{*}{ Sheathia arcuata } & GU457344 & D 保加利亚 & {$[47]$} \\
\hline & JN086522 & E 中国 & {$[48]$} \\
\hline
\end{tabular}




\section{2 研究结果}

\section{1 分子鉴定与系统发育分析}

2.1.1 序列特征分析 所测 3 条序列上传至 GenBank 数据库, 所得序列登录号分别为 $r b c \mathrm{~L}-\mathrm{MH} 220972 、 \mathrm{COI}-$ MH220973 和 UPA-MH220974, 长度分别为 1203 、686 和 357 bp. 3 个序列与 GenBank 数据库在线比对结果显 示, JC1712001 与熊野藻属及串珠藻属的物种序列比对率较高, 使用 Clustal 软件对所有序列进行比对, 并用 MEGA 6.0 分析序列特征, 经比对校正后 $r b c \mathrm{~L} 、 \mathrm{COI}$ 和 UPA 基因的序列长度分别为 1203 、661 和 $319 \mathrm{bp}$, 其中 核苷酸变异位点分别为 496、335 和 107 个,简约信息位点分别为 $434 、 305$ 和 90 个,3 个基因都与马赫拉熊野 藻 (Kumanoa mahlacensis Kumano) 遗传距离最近,均为 0 .

利用 Modeltest 3.7 软件对序列最适合的进化模型进行分析, 获得合适的进化模型, 并得到相关模型参数 (表3).

表 3 Modeltest 3.7 分析获得的 $r b c \mathrm{~L} 、 \mathrm{COI}$ 和 UPA 基因模型及相关参数

Tab.3 Nucleotide substitution model parameter estimates of $r b c \mathrm{~L}$, COI and UPA gene for Modeltest 3.7 analyses

\begin{tabular}{|c|c|c|c|}
\hline 基因类型 & 模型参数 & 碱基频率 & 矩阵参数 \\
\hline \multirow[t]{6}{*}{$r b c \mathrm{~L}$} & $\mathrm{GTR}+\mathrm{I}+\mathrm{G}-\ln \mathrm{L}=10562.1104$ & freq $A=0.3718$ & $\mathrm{R}(\mathrm{a})[\mathrm{A}-\mathrm{C}]=1.7976$ \\
\hline & $K=10$ & freqC $=0.1181$ & $\mathrm{R}(\mathrm{b})[\mathrm{A}-\mathrm{G}]=5.0097$ \\
\hline & $(\mathrm{I})=0.4931$ & freqG $=0.1370$ & $\mathrm{R}(\mathrm{c})[\mathrm{A}-\mathrm{T}]=0.4997$ \\
\hline & $(G)=0.7465$ & freq $\mathrm{T}=0.3731$ & $\mathrm{R}(\mathrm{d})[\mathrm{C}-\mathrm{G}]=1.4924$ \\
\hline & & & $\mathrm{R}(\mathrm{e})[\mathrm{C}-\mathrm{T}]=9.9703$ \\
\hline & & & $\mathrm{R}(\mathrm{f})[\mathrm{G}-\mathrm{T}]=1.0000$ \\
\hline \multirow[t]{6}{*}{ COI } & $\mathrm{TVM}+\mathrm{I}+\mathrm{G}-\ln \mathrm{L}=7759.7026$ & freq $A=0.3052$ & $\mathrm{R}(\mathrm{a})[\mathrm{A}-\mathrm{C}]=1.5388$ \\
\hline & $K=9$ & freqC $=0.1119$ & $\mathrm{R}(\mathrm{b})[\mathrm{A}-\mathrm{G}]=12.2582$ \\
\hline & $(\mathrm{I})=0.4116$ & freqG $=0.0978$ & $\mathrm{R}(\mathrm{c})[\mathrm{A}-\mathrm{T}]=0.3658$ \\
\hline & $(G)=0.6293$ & freq $\mathrm{T}=0.4851$ & $\mathrm{R}(\mathrm{d})[\mathrm{C}-\mathrm{G}]=2.8124$ \\
\hline & & & $\mathrm{R}(\mathrm{e})[\mathrm{C}-\mathrm{T}]=12.2582$ \\
\hline & & & $R(f)[G-T]=1.0000$ \\
\hline \multirow[t]{6}{*}{ UPA } & $\operatorname{TrN}+\mathrm{I}+\mathrm{G}-\ln \mathrm{l}=1850.3889$ & freq $A=0.2790$ & $\mathrm{R}(\mathrm{a})[\mathrm{A}-\mathrm{C}]=1.0000$ \\
\hline & $K=7$ & freqC $=0.1685$ & $\mathrm{R}(\mathrm{b})[\mathrm{A}-\mathrm{G}]=5.4551$ \\
\hline & $(\mathrm{I})=0.3999$ & freqG $=0.2706$ & $\mathrm{R}(\mathrm{c})[\mathrm{A}-\mathrm{T}]=1.0000$ \\
\hline & $(G)=0.5816$ & freq $\mathrm{T}=0.2819$ & $\mathrm{R}(\mathrm{d})[\mathrm{C}-\mathrm{G}]=1.0000$ \\
\hline & & & $\mathrm{R}(\mathrm{e})[\mathrm{C}-\mathrm{T}]=10.4471$ \\
\hline & & & $\mathrm{R}(\mathrm{f})[\mathrm{G}-\mathrm{T}]=1.0000$ \\
\hline
\end{tabular}

2.1.2 基于 $r b c \mathrm{~L} 、 \mathrm{COI}$ 和 UPA 基因的系统进化分析 以研究藻株 JC1712001 与 GenBank 中下载的 30 多个串 珠藻科物种为研究对象, 以红毛菜 Bangia atropurpurea (Mertens ex Roth) Agardh C 和 B. fuscopurpurea Holmes $\&$ Batters 为外类群, 基于 $r b c \mathrm{~L} 、 \mathrm{COI}$ 和 UPA 基因序列, 分别采用邻接法、贝叶斯法和最大似然法构建系统发 育树的结果表明, 基于 3 种方法得到的系统树具有高度统一的拓扑结构, 以贝叶斯树为基础, 将邻接法、贝 叶斯法和最大似然法所得到的支持率分别标记于分支节点处(图 1、2 和 3).

从基于 $r b c \mathrm{~L}$ 基因构建的系统树可看出, JC1712001 与产于西班牙 (JQ713334) 和美国 ( JN590006、 JN590007）的马赫拉熊野藻聚为一支,3 种方法的支持率均为 100\%. 以 COI 基因构建的系统树也反映出, JC1712001 与产于美国 (JN604905、JN604906) 的马赫拉熊野藻聚为一支, 且 3 种方法的支持率也均为 100\%. 以 UPA 基因构建的系统树也显示了同样的结果, JC1712001 与产于美国 (JN602660、JN602661) 的马赫拉熊 野藻聚为一支, 3 种方法的支持率分别为 97\%、97\% 和 $100 \%$.

由 3 个基因构建的系统发育研究结果均支持 JC1712001 为马赫拉熊野藻. 


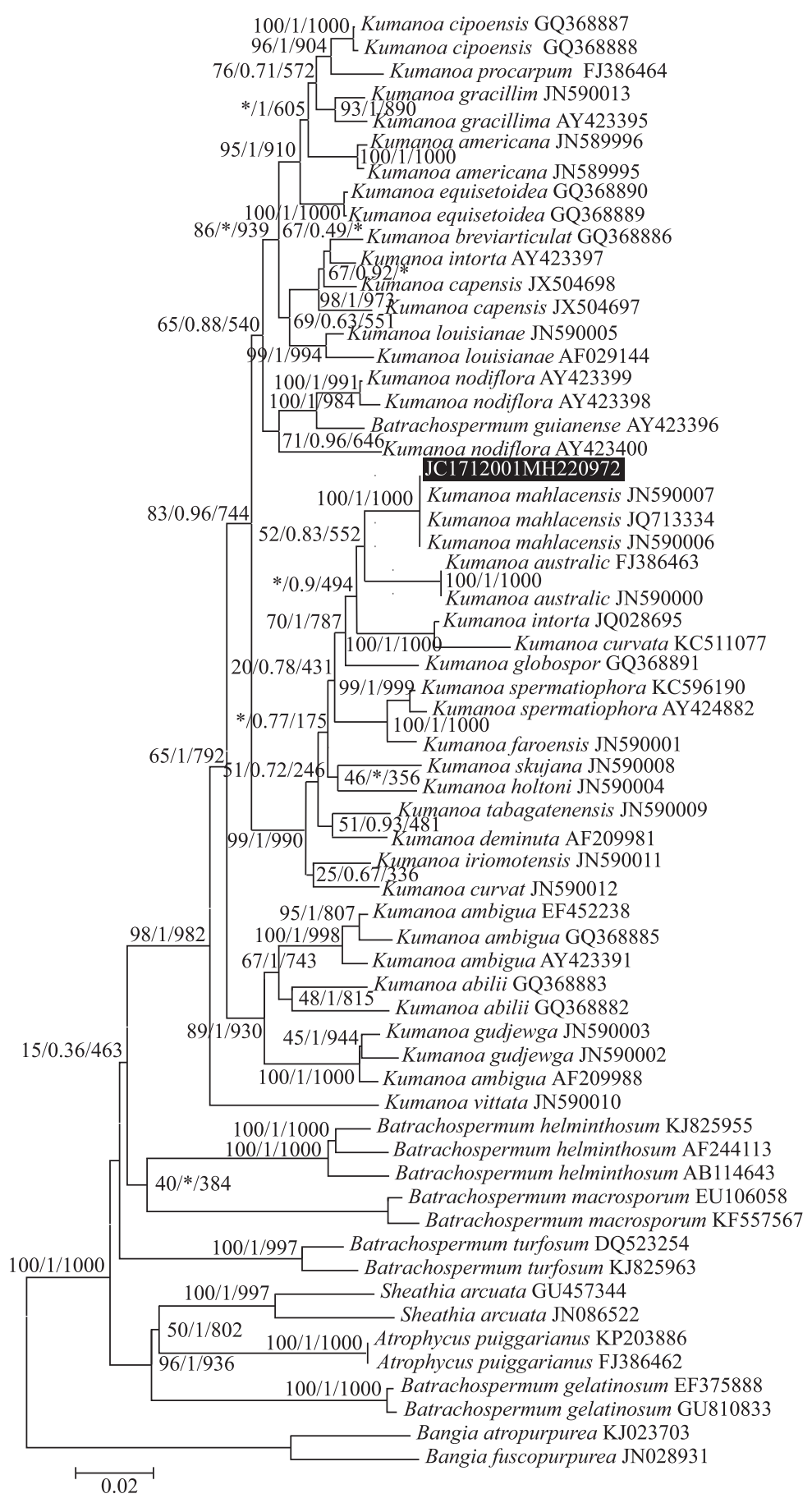

图 1 基于 $r b c \mathrm{~L}$ 基因序列的系统树(各分支数字分别代表 $\mathrm{NJ}$ 法步靴值、 BA 后验概率和 ML 法步靴值, “*”表示该方法结果不支持该聚类分支)

Fig.1 Bayesian analysis tree based on the $r b c \mathrm{~L}$ gene sequence (Support values for all analyses are shown as follows: NJ distance bootstrap/Bayesian posterior probabilities/ML bootstrap; ' $*$ ' denotes no support for that analyses at that node) 


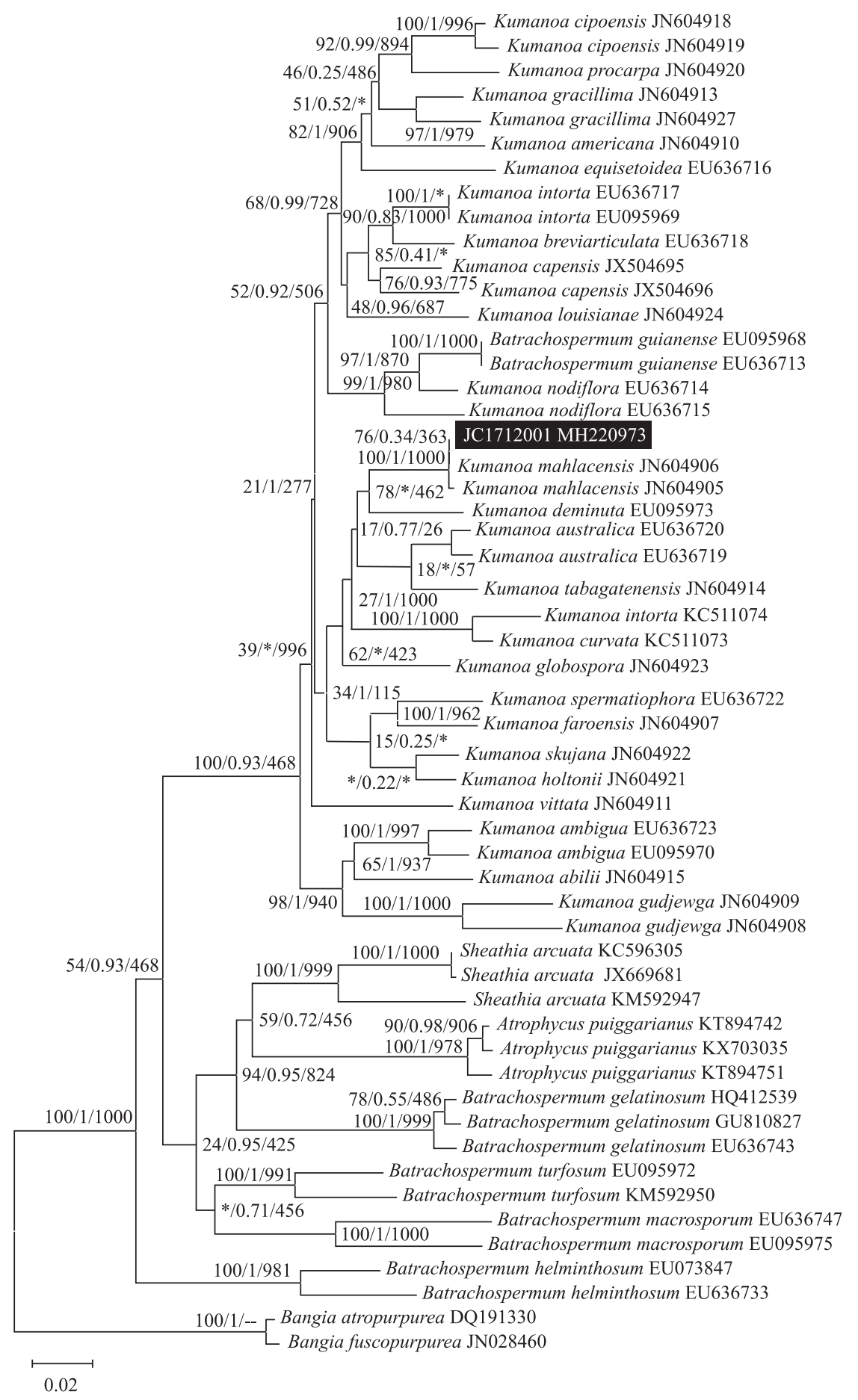

图 2 基于 COI 基因序列的系统树(各分支数字分别代表 NJ 法步靴值、

BA 后验概率和 ML 法步靴值, “*”表示该方法结果不支持该聚类分支)

Fig.2 Bayesian analysis tree based on the COI gene sequence (Support values for all analyses are shown as follows: NJ distance bootstrap/Bayesian posterior probabilities/ML bootstrap; ' *' denotes no support for that analyses at that node) 


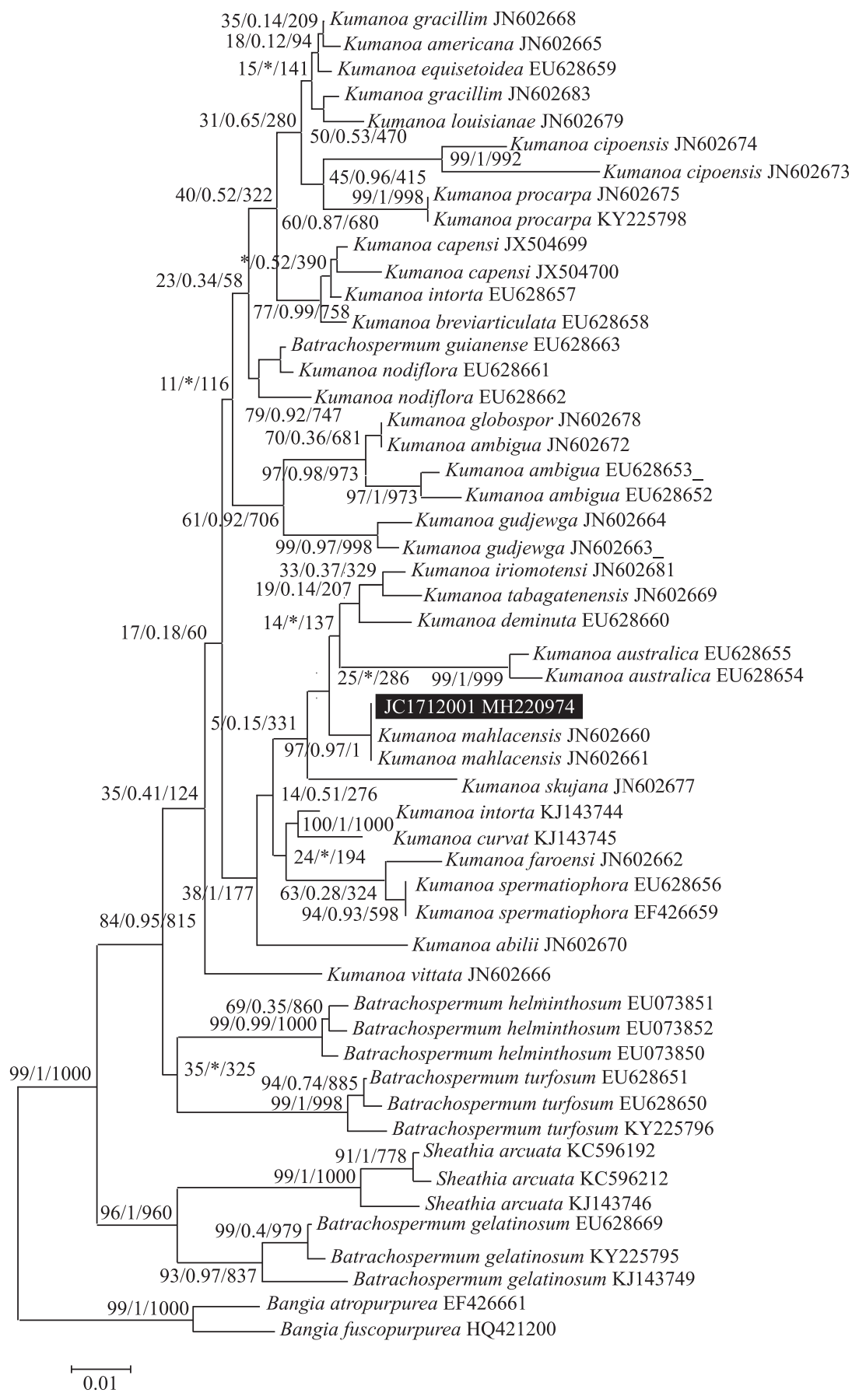

图 3 基于 UPA 基因序列的系统树(各分支数字分别代表 NJ 法步靴值、 BA 后验概率和 ML 法步靴值, “*”表示该方法结果不支持该聚类分支)

Fig.3 Bayesian analysis tree based on the UPA gene sequence (Support values for all analyses are shown as follows: NJ distance bootstrap/Bayesian posterior probabilities/ML bootstrap; ' $*$ ' denotes no support for that analyses at that node) 


\section{2 形态观察结果}

JC1712001 形态观察结果见图 4. 藻体橄榄绿色, 丛生, 胶质较少, 高 5 12 cm, 雌雄同株 (图 4A). 轮节 为梨形, 直径 250 400 $\mu \mathrm{m}$, 初生枝 2 次分叉, 由 $6 \sim 8$ 层细胞组成, 下部细胞长卵形, 长 $10 \sim 20 \mu \mathrm{m}$, 直径约 8 $\mu \mathrm{m}$, 顶毛多, 较长, 达 $200 \mu \mathrm{m}$. 皮层细胞发达, 长筒状, 长 $18 \sim 27 \mu \mathrm{m}$, 直径 $7 \sim 8 \mu \mathrm{m}$, 次生枝布满节间, $1 \sim 10$ 个细胞长, 不分叉, 细胞较初生枝小 (图 4B). 果孢子体每轮节 1 个, 紧密, 半球形, 紧贴主轴, 直径 $120 \sim 150$ $\mu \mathrm{m}$, 高 100 120 $\mu \mathrm{m}$, 果孢子囊倒卵形, 长 10 13 $\mu \mathrm{m}$, 直径 7 8 $\mu \mathrm{m}$ (图 4C). 果孢枝发生于初生枝基部, 螺旋 状扭曲, 由 $6 \sim 8$ 个盘形细胞组成, 细胞长 5 8 $\mu \mathrm{m}$, 直径 6 9 $\mu \mathrm{m}$, 受精丝筒状棒形, 无明显的柄 (图 4D). 精 子囊球形,单生或双生,位于初生枝顶端,直径 4 8 $\mu \mathrm{m}$ (图 4E).

从形态观察结果看, JC1712001 具有典型的马赫拉熊野藻形态特点, 同样支持其为马赫拉熊野藻. 从表 4 也可看出, JC1712001 与之前报道过的不同地区的马赫拉熊野藻形态近似.
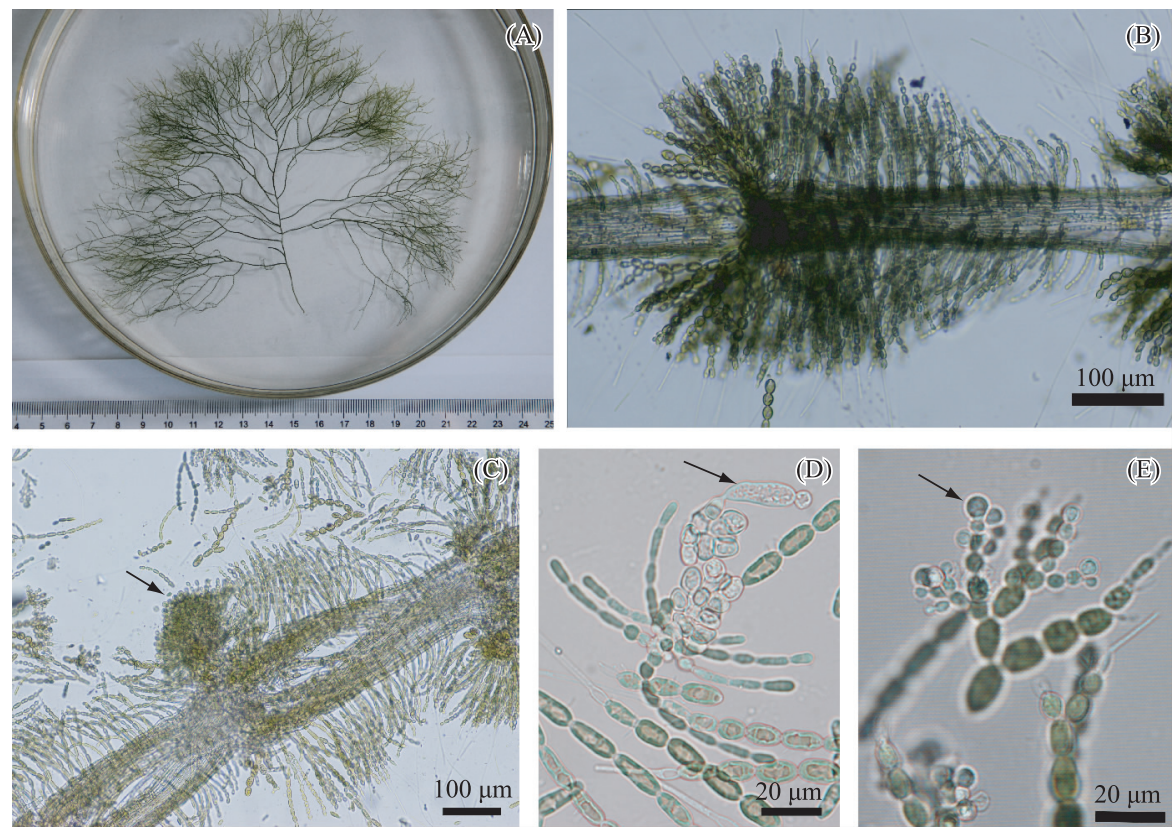

图 4 马赫拉熊野藻 Kumanoa mahlacensis Kumano 整体及显微照片 ( $\mathrm{A}$ : 藻体外形 $; \mathrm{B}$ : 轮节; $\mathrm{C}$ : 果孢子体 (箭头) ; $\mathrm{D}$ : 果胞及受精丝 (箭头) $; \mathrm{E}$ : 精子囊 (箭头))

Fig.4 Habit and microphotographs of Kumanoa mahlacensis

( A: the habit of frond; B: whorls; C: carposporophyte(arrows);

D: carpogonia and trichogune (arrows) ; E: spermatangia (arrows $)$ )

\section{3 地理起源分析}

选取包括 1 个外类群和串珠藻科 34 个种不同地理位置的 48 条 $r b c \mathrm{~L}$ 序列, 构建的祖先地理分布情况见 图 5. 由图 5 可看出,所选样本的地理分布特点为南美洲 18 个、北美洲 11 个、大洋洲 6 个、欧洲 5 个、亚洲 6 个、太平洋岛国 1 个、非洲 1 个. 马赫拉熊野藻在欧洲、亚洲、北美洲均有分布, 欧洲类群地理起源更为古老.

\section{3 讨论}

基于 $r b c \mathrm{~L} 、 \mathrm{COI}$ 和 UPA 基因序列构建的系统发育树均表明,采自山西晋祠泉的串珠藻状植物 JC1712001 与西班牙及美国地区已报道的马赫拉熊野藻聚为一支, 支持率都在 $97 \%$ 以上, 可以确定其为马赫拉熊野藻, 同时基于形态学比较, 该澡株与其他产地的该种马赫拉熊野藻形态相似, 具有典型的马赫拉熊野澡特征: 果 孢枝螺旋状扭曲, 受精丝筒状棒形, 无明显的柄, 次生枝多, 布满节间 ${ }^{[45,49-51]}$, 与分子鉴定结果一致. 
表 4 不同地区马赫拉熊野藻 Kumanoa mahlacensis 形态比较

Tab.4 Morphological comparison of Kumanoa mahlacensis in different regions

\begin{tabular}{|c|c|c|c|c|c|}
\hline 形态特征 & 关岛 (美国) & 泰国 & 西班牙 & 四川( 中国) & 山西( 中国) \\
\hline 藻体高度/cm & - & $2 \sim 6$ & 6 & 2 & $6 \sim 12$ \\
\hline 轮节形状 & - & 梨形 & 梨形 & 截顶雉形 & 梨形 \\
\hline 轮节直径/ $\mu \mathrm{m}$ & - & $250 \sim 400$ & $220 \sim 340$ & $250 \sim 350$ & $250 \sim 400$ \\
\hline 节间长度和宽度/ $\mu \mathrm{m}$ & - & $200 \sim 400$ & - & $230 \sim 400$ & $300 \sim 400$ \\
\hline 皮层丝细胞长度和直径/ $\mu \mathrm{m}$ & - & - & - & $27 \sim 26,7 \sim 8$ & $18 \sim 27,7 \sim 8$ \\
\hline 初生枝细胞长度/cells & $7 \sim 9$ & $7 \sim 9$ & $7 \sim 9$ & $6 \sim 8$ & $6 \sim 8$ \\
\hline 次生枝长度/cells & $6 \sim 7$ & $6 \sim 7$ & $6 \sim 7$ & $1 \sim 6$ & $3 \sim 10$ \\
\hline 果胞枝长度/cells & $5 \sim 15$ & $5 \sim 15$ & $5 \sim 15$ & $4 \sim 9$ & $7 \sim 10$ \\
\hline 果胞长度和直径/ $\mu \mathrm{m}$ & $25 \sim 40,4 \sim 5$ & $25 \sim 40,4 \sim 5$ & $15 \sim 25,4 \sim 5$ & $30 \sim 40,6 \sim 10$ & $25 \sim 40,7 \sim 8$ \\
\hline 果孢子体直径和高度/ $\mu \mathrm{m}$ & $140 \sim 170,-$ & $140 \sim 170,80 \sim 160$ & $125 \sim 155,-$ & $200 \sim 250,120 \sim 150$ & $120 \sim 150,100 \sim 120$ \\
\hline 果孢子囊直径和长度/ $\mu \mathrm{m}$ & - & - & - & $7 \sim 8,11 \sim 13$ & $6 \sim 7,10 \sim 13$ \\
\hline 精子囊直径/ $\mu \mathrm{m}$ & - & $4 \sim 6$ & $4 \sim 6$ & $5 \sim 6$ & $4 \sim 8$ \\
\hline 参考文献 & {$[49]$} & {$[50]$} & {$[44]$} & {$[51]$} & 本研究 \\
\hline
\end{tabular}

1998-2005 年, Vis 等基于 $r b c \mathrm{~L}$ 和 SSU 序列先后对采自澳大利亚和法属硅亚那的串珠藻目样本进行研 究, 结果均表明串珠藻属 Batrachospermum 的杂生组 Section Hybrida 和扭曲组 Section Contorta 物种具有较近 的亲缘关系, 且具有相似的形态学特征,果胞枝明显弯曲、扭曲或螺旋状 ${ }^{[21,32,41]} .2009$ 年, Entwisle 等将杂生 组和扭曲组从串珠藻属中分离出来建立了熊野藻属 ${ }^{[3]}$. 2015 年, 南芳茹等基于叶绿体 $p s a \mathrm{~A}$ 和 $p s b \mathrm{~A}$ 基因对 中国熊野藻属植物系统发育进行研究, 结果支持该属的建立 ${ }^{[20]}$. 目前, 中国已报道熊野藻属物种共 10 种 ${ }^{[20]}$. 相关分子数据研究十分薄弱, 仅限于 2 种, 即弯形熊野藻 $K$. curvata Shi 和绞扭熊野藻 $K$. intorta $\mathrm{Jao}^{[20,42-43,45]}$

马赫拉熊野藻于 1986 年由 Kumano 和 Bowden-Kerby 建立,模式标本在太平洋岛屿关岛首次发现, 目前 包括中国在内仅有 4 个国家和地区有过报道, 为稀有种, 分子数据也仅报道 8 条 ${ }^{[44,50]}$. 本文的研究结果将丰 富该种的分布和分子数据.

研究结果还显示产自不同地区的马赫拉熊野藻的 $r b c \mathrm{~L} 、 \mathrm{COI}$ 和 UPA 基因序列具有高度的相似性, 3 个产 地的样本遗传距离为 0.00 , 说明 3 个基因在种内具有高度的保守性. 基于 3 个基因的系统树拓扑结构相似， 熊野藻属物种均聚在一支且与其他属所在分支相分离, 支持熊野藻属的建立. 从进化树还可以看出 3 个基 因在种间和属间具有明显差异, 说明 $r b c \mathrm{~L} 、 \mathrm{COI}$ 和 UPA 基因序列能够反映熊野藻属等的系统发育关系, 可以 作为其系统发育研究的分子标记. 从系统发育树可以看到, 我国境内分布的 3 个熊野藻属种类亲缘关系较 远,各自位于不同的进化支,而山西晋祠的马赫拉熊野藻与 $K$. australica Entwisle \& Foard 和 K. deminuta Entwisle \& Foard (采自大洋洲与南美洲) 遗传距离较近, 聚类在进化树的同一分支上, 可见熊野藻属起源较早, 经历了漫长的进化历史, 形成现今的同种全球广泛分布模式, 这与其他关于熊野藻属的系统进化研究相 一致 ${ }^{[3,40,43]}$.

地理起源分析显示,马赫拉熊野藻在欧洲、亚洲、北美洲均有分布, 欧洲类群地理起源更为古老,这与熊 野藻属的地理起源一致, 其祖先分布区节点在欧洲, 并向大洋洲、北美洲、南美洲扩散,这也与与南芳茹等基 等基于 UPA 序列的熊野藻属地理起源分析中对熊野藻属的地理分布描述一致 ${ }^{[43]}$. 谢树莲和凌元洁研究了 串珠藻目的现代地理起源和分布, 对串珠藻可能的散布途径做出解释, 串珠藻目起源于古地中海沿岸, 然后 向外辐射, 向欧亚大陆扩展, 随后成为串珠藻目的现代起源中心, 大洋洲和北美洲是两个次级分布中心 ${ }^{[52]}$. 结合本研究的结果, 可初步分析得出, 早期的串珠藻起源于欧洲, 从欧洲向大洋洲、北美洲和南美洲扩散的 过程中, 一方面直接向各大洲扩散迁移(集中分布在欧洲和亚洲, 在各大洲均有分布), 另一方面由于地质巨 变和气候变迁的影响, 逐步向熊野藻属演变( 随着扩散的发生熊野藻属的物种越来越多,位于进化末端的美 洲地区熊野藻属大部分为地区特有种). 但鉴于目前化石证据较少, 供研究的样本也还有限, 未见报道的地 

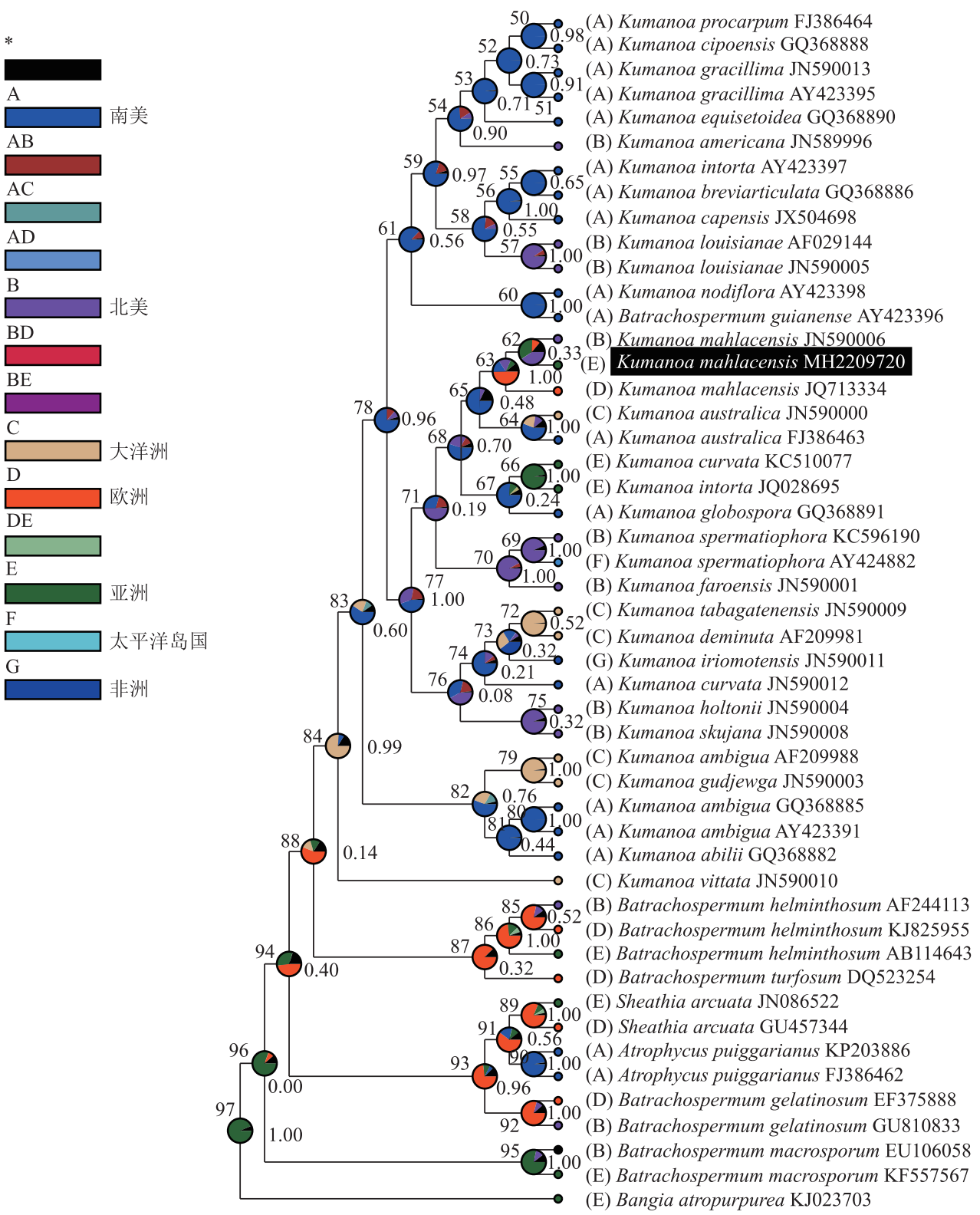

图 5 基于 $r b c \mathrm{~L}$ 序列的祖先地理分布

Fig.5 Ancestor geographical map based on $r b c \mathrm{~L}$ sequences

区,并不一定就没有分布,分析结果还具有很大的局限性,仍有待于进一步深人研究.

\section{4 结论}

1) 基于 $r b c \mathrm{~L} 、 \mathrm{COI}$ 和 UPA 序列的分子系统发育分析表明,山西晋祠泉采集的的串珠藻状植物 JC1712001 为马赫拉熊野藻 Kumanoa mahlensis Kumano, 为山西省新纪录种.

2) JC1712001 具有典型的马赫拉熊野藻形态特点, 与之前报道过的不同地区的马赫拉熊野藻形态近似, 同样支持其为马赫拉熊野藻. 
3) 地理起源分析结果显示, 马赫拉熊野藻在欧洲、亚洲、北美洲均有分布, 欧洲类群地理起源更为古老, 与熊野藻属的地理起源一致.

\section{5 参考文献}

[ 1 ] Yang EC, Boo SM, Bhattacharya D et al. Divergence time estimates and the evolution of major lineages in the florideophyte red algae. Scientific Reports, 2016, 6: 21361.

[ 2 ] Kumano S. Freshwater red algae of the world. Journal of Phycology, 2002, 38: 834-835.

[ 3 ] Entwisle TJ, Vis ML, Chiasson WB et al. Systematics of the Batrachospermales (Rhodophyta) - a synthesis. Journal of Phycology, 2009, 45: 704-715.

[ 4 ] Agardh CA ed. Systema Algarum. Lund, 1824: 1-312.

[ 5 ] Roth AW ed. Bemerkungen über das Studium der Cryptogamischen Wassergewächse. Hannover: Gebrüdern, 1797 : 109.

[ 6 ] Nees CG ed. Horae physicae Berolinenses collectae ex symbolis virorum doctorum. Bonnae: Sumtibus Adolphi Marcus, 1820: $1-123$.

[ 7 ] Bonnemaison T ed. Essai sur les hydrophytes loculées (ou articulées) de la famille des Épidermées et des Céramiées. Paris: Mémoires du Muséum d’Histoire Naturelle, 1828, 16: 49-148.

[ 8 ] Rossignolo NL, Necchi OJr. Revision of section Setacea of the genus Batrachospermum (Batrachospermales, Rhodophyta) with emphasis on specimens from Brazil. Phycologia, 2016, 55: 337-346.

[ 9 ] Harvey WH. Nereis Boreali-Americana: or contributions to a history of the marine Algae of North America. Smithsonian Contributions to Knowledge, 1858, 10: 37-50.

[10] Kylin H. Studien über die Schwedischen Arten der Gattungen Batrachospermum Roth und Sirodotia nov. gen. Nova Acta Regiae Soc Sci Upsaliensis, Ser. IV, 1912, 3: 1-40.

[11] Skuja H. Untersuchungen über die Rhodophyceen des Sußwassers. Beihefte zum Botanische Centralblatt, 1934, 52 : 173-192.

[12] Entwisle TJ. Psilosiphon scoparium gen. et sp. nov. (Lemaneaceae), a new red alga from south-eastern Australian streams. Phycologia, 1989, 28: 469-475.

[13] Saunders GW, Necchi OJr. Nuclear rDNA sequences from Ballia prieurii support recognition of Balliopsis gen. nov. in the Batrachospermales (Florideophyceae, Rhodophyta). Phycologia, 2002, 41: 61-67.

[14] Vis ML, Harper JT, Saunders GW. Large subunit rDNA and $r b c \mathrm{~L}$ gene sequence data place Petrohua bernabei gen. et sp. nov. in the Batrachospermales (Rhodophyta), but do not provide further resolution among taxa in this order. Phycological Research, 2007, 55: 103-112.

[15] Salomaki ED, Wandrans JK, Eloranta P et al. Molecular and morphological evidence for Sheathia gen. nov. ( Batrachospermales, Rhodophyta) and three new species. Journal of Phycology, 2014, 50: 526-542.

[16] Rossignolo NL, Necchi OJr, Guiry MD. Atrophycus, a new genus name for “Setacea (De Toni) Necchi and Rossignolo". Notulae Algarum, 2017, 26: 1-2.

[17] Entwisle TJ, Johnston ET, Lam DW et al. Nocturama gen. nov, Nothocladus s. lat. and other taxonomic novelties resulting from the further resolution of paraphyly in Australasian members of Batrachospermum (Batrachospermales, Rhodophyta). Journal of Phycology, 2016, 52: 384-396.

[18] Evans JR, Chapuis IS, Vis ML. Adding to the freshwater red algal diversity in North America: Lympha mucosa gen. et sp. nov. (Batrachospermales, Rhodophyta). Algae, 2017, 32: 171-179.

[19] Chapuis IS, Necchi OJr, Zuccarello GC et al. A new genus, Volatus and four new species of Batrachospermum sensu stricto (Batrachospermales, Rhodophyta). Phycologia, 2017, 56: 454-468.

[20] Nan FR, Feng J, Xie SL. Phylogenetic relationship of genus Kumanoa based on chloroplaet psaA and psbA genes. Acta Hydrobiologica Sinica, 2015, 39: 155-16. [南芳茹, 冯佳, 谢树莲. 基于叶绿体 $p s a \mathrm{~A}$ 和 $p s b \mathrm{~A}$ 基因的中国熊野藻属 植物系统发育分析. 水生生物学报, 2015, 39: 155-161.]

[21] Vis ML, Saunders GW, Sheath RG et al. Phylogeny of the Batrachospermales (Rhodophyta) inferred from $r b c \mathrm{~L}$ and $18 \mathrm{~S}$ ribosomal DNA gene sequences. Journal of Phycology, 1998, 34: 341-350.

[22] Saunders GW. Applying DNA barcoding to red macroalgae: a preliminary appraisal holds promise for future applications. 
Philosophical Transactions of the Royal Society of London, 2005, 360: 1879-1888.

[23] Sherwood AR, Presting GG. Universal primers amplify a 23S rDNA plastid marker in eukaryotic algae and cyanobacteria. Journal of Phycology, 2007, 43: 605-608.

[24] Necchi OJr, Vis ML, Oliveira MC. Phylogenetic relationships in Kumanoa (Batrachospermales, Rhodophyta) species in Brazil with the proposal of Kumanoa amazonensis sp nov. Phycologia, 2010, 49: 97-103.

[25] Deng YY, Lu QQ, Shen DS et al. Cytological observations and $r b c \mathrm{~L}$, nrSSU gene sequence analyses of filamentous Bangiales (Rhodophyta) from China. Journal of Applied Phycology, 2015, 27: 1355-1363.

[26] Kucera H, Saunders GW. A survey of bangiales (rhodophyta) based on multiple molecular markers reveals cryptic diversity. Journal of Phycology, 2012, 48: 869-882.

[27] Sherwood AR, Kurihara A, Conklin KY et al. The Hawaiian Rhodophyta Biodiversity Survey (2006-2010): a summary of principal findings. BMC Plant Biology, 2010, 10: 258.

[28] House DL, Vandenbroek AM, Vis ML. Intraspecific genetic variation of Batrachospermum gelatinosum (Batrachospermales, Rhodophyta) in eastern North America. Phycologia, 2010, 49: 501-507.

[29] Stewart SA, Vis ML. Investigation of two species complexes in Batrachospermum section Batrachospermum (Batrachospermales, Rhodophyta). Phycologia, 2007, 46: 380-385.

[30] House DL, Sherwood AR, Vis ML. Comparison of three organelle markers for phylogeographic inference in Batrachospermum helminthosum (Batrachospermales, Rhodophyta) from North America. Phycological Research, 2008, 56: 69-75.

[31] Rueness J. DNA barcoding of select freshwater and marine red algae (Rhodophyta). Cryptogamie Algologie, 2010, 31: 377-386.

[32] Vis ML, Chiasson WB, Sheath RG. Phylogenetic relationship of Batrachospermum species (Batrachospermales, Rhodophyta) from coastal streams in French Guiana. Phycologia, 2005, 44: 441-446.

[33] Sherwood AR, Vis ML, Entwisle TJ et al. Contrasting intra versus interspecies DNA sequence variation for representatives of the Batrachospermales (Rhodophyta): Insights from a DNA barcoding approach. Phycological Research, 2008, 56: 269-279.

[34] Chiasson WB, Salomaki ED, Vis ML. New collections of freshwater red algae (Batrachospermales, Rhodophyta) from historically important areas in France. Cryptogamie Algologie, 2014, 35: 303-316.

[35] Hanyuda T, Suzawa Y, Suzawa T et al. Biogeography and taxonomy of Batrachospermum helminthosum ( Batrachospermales, Rhodophyta) in Japan inferred from $r b c \mathrm{~L}$ gene sequences. Journal of Phycology, 2004, 40: 581-588.

[36] Chiasson WB, Machesky NJ, Vis ML. Phylogeography of Batrachospermum Helminthosum (Rhodophyta) in North America. Journal of Phycology, 2010, 38 ( s1) : 3-4.

[37] Vis ML, Hodge JC, Necchi OJr. Phylogeography of Batrachospermum macrosporum (Batrachospermales, Rhodophyta) from North and South America. Journal of Phycology, 2008, 44: 882-888.

[38] Johnston ET, Lim PE, Buhari N et al. Diversity of freshwater red algae (Rhodophyta) in Malaysia and Indonesia from morphological and molecular data. Phycologia, 2014, 53: 329-341.

[39] Kapraun DF, Braly KS, Freshwater DW. Nuclear DNA content variation in the freshwater red algal orders Batrachospermales and Thoreales. Phycologia, 2007, 46: 54-62.

[40] Vis ML, Necchi OJr, Chiasson WB et al. Molecular phylogeny of the genus Kumanoa (Batrachospermales, Rhodophyta). Journal of Phycology, 2012, 48: 750-758.

[41] Vis ML, Entwisle TJ. Insights into the phylogeny of the Batrachospermales (Rhodophyta) from $r b c L$ sequence data of Australian taxa. Journal of Phycology, 2000, 36: 1175-1182.

[42] Ji L, Xie SL, Feng J et al. Molecular systematics of the four endemic Batrachospermaceae (Rhodophyta) species in China with multilocus data. Journal of Systematics and Evolution, 2014, 52: 92-100.

[43] Nan FR, Feng J, Xie SL. Phylogenetic relationship of genus Kumanoa (Batrachospermales, Rhodophyta) based on UPA genes. Bull Bot Res, 2014, 34: 584-591. [南芳茹, 冯佳, 谢树莲. 中国熊野藻属植物系统发育分析一一基于叶绿 体 UPA 序列. 植物研究, 2014, 34: 584-591.]

[44] Garcia-fernandez M, Vis ML, Aboal M. Kumanoa mahlacensis (Batrachospermales, Rhodophyta) in a Mediterranean coastal wetland, a new species for the European continental algal flora. Anales Del Jardin Botanico De Madrid, 2015,72 (1): e018. 
[45] Li Q, Ji L, Xie SL. Phylogenetic analysis of Batrachospemales (Florideophyceae, Rhodophyta) based on chloroplast $r b c \mathrm{~L}$ sequences. Acta Hydrobiologica Sinica, 2010, 34: 20-28. [李强, 吉莉, 谢树莲. 串珠藻目植物的系统发育一一基于 $r b c \mathrm{~L}$ 序列的证据. 水生生物学报, 2010, 34: 20-28.]

[46] Carlile AL, Sherwood AR. Phylogenetic affinities and distribution of the Hawaiian freshwater red algae (Rhodophyta). Phycologia, 2013, 52: 309-319.

[47] Vis ML, Feng J, Chiasson WB et al. Investigation of the molecular and morphological variability in Batrachospermum arcuatum (Batrachospermales, Rhodophyta) from geographically distant locations. Phycologia, 2010, 49: 545-553.

[48] Ji L, Feng J, Chen L et al. A molecular and morphological investigation of Batrachospermum arcuatum (Batrachospermales, Rhodophyta) in China. Aquatic Botany, 2011, 95: 254-257.

[49] Kumano S, Bowden-Kerby WA. Studies of the freshwater Rhodophyta of Micronesia I. Six new species of Batrachospermum Roth. Japanese Journal of Phycology, 1986, 34: 107-128.

[50] Traichaiyaporn S, Kumano S, Chainapong T. New record of freshwater red algae (Rhodophyta) in Thailand Batrachospermum mahlacense Kumano et Boden-Kerby. Science Asia, 2004, 30: 313-315.

[51] Xie SL, Shi ZX, Lu HS et al. Four new reports of Batrachospermum (Batrachospermales, Rhodophyta) from China. Journal of Wuhan Botanical Research, 2003, 21: 148-152. [谢树莲, 施之新, 卢海声等. 中国串珠藻属的 4 个新记录种. 武汉植物学研究, 2003, 21: 148-152.]

[52] Xie SL, Ling YJ. Modern geographical distribution and origin of Batrachospermales. Journal of Shanxi University: Nat Sci $E d, 2002,25: 149-155$. [谢树莲, 凌元洁. 串珠藻目的现代地理分布和起源. 山西大学学报: 自然科学版, 2002, 25: $149-155$. ] 